\title{
Raman spectroscopy and its use for live cell and tissue analysis
}

\author{
Hidetoshi Sato $^{\mathrm{a}, *}$, Mika Ishigaki ${ }^{\mathrm{b}}$, Akinori Taketani ${ }^{\mathrm{a}}$ and Bibin B. Andriana ${ }^{\mathrm{a}}$ \\ ${ }^{a}$ Department of Biomedical Chemistry, School of Science and Technology, Kwansei Gakuin University, \\ 2-1, Gakuen, Sanda, Hyogo 669-1337, Japan \\ ${ }^{\mathrm{b}}$ Center for the Promotion of Project Research, Organization for Research and Academic Information, \\ and Faculty of Life and Environmental Science, Shimane University, 1060 Nishikawatsu-cho, \\ Matsue-shi, Shimane 690-8504, Japan
}

\begin{abstract}
As research progresses in the field of life sciences, there is an increased demand for new technologies that can allow us to study intact cells and tissues. The quantitative analysis and mathematical modeling of living things based on empirical data is useful for connecting molecular biology to new areas, such as computational biology. Raman spectroscopy is regarded as one of the possible methods by which we can observe living organisms in a noninvasive manner. This could improve the quality of research in the field of medicine and health and will largely contribute to society in the future. The present review introduces some techniques based on Raman spectroscopy and evaluates their applications in intact live samples.
\end{abstract}

Keywords: Raman spectroscopy, Biomedical, Raman probe, in vivo

\section{Introduction}

It is very interesting to explore life by observing the activity of living cells and tissues. The Nobel Prize in Chemistry was awarded to Shimomura, Chalfie, and Tsien in 2008 for their discovery and development of fluorescent proteins [21,22]. Such proteins permits the observation of protein localization and migration in live materials by using fluorescence. This development has greatly contributed to the understanding molecular kinetics. However, it is not a perfect technique. The fluorescent protein often disturbs the localization and migration of the targeted protein, which may be fatal, especially for animal samples. It is not applicable for use in human studies because it would require gene modification. Hence, to our knowledge, the only technique used to observe molecular activity in humans is Raman spectroscopy.

Raman spectroscopy is one of the vibrational spectroscopies which includes infrared (IR) and nearinfrared (NIR) spectroscopies as well [13,23]. Since the vibrational spectroscopy is based on the transition between vibrational energy states of molecules, all molecules produce a signal for observation by IR or Raman spectroscopy. IR spectroscopy is an absorption spectroscopy method in which a molecule absorbs light with a specific wavenumber. Raman spectroscopy is a scattering method in which scattered light with specific wavenumbers are observed. The vibrational energy transition is generally permitted only between neighboring energy states, and this generates IR and Raman signals. A molecule consisting of "N" atoms has "3N-6" ("3N-5" for linear molecules) vibrational modes. Since each vibrational

\footnotetext{
${ }^{*}$ Corresponding author. E-mail: hidesato@kwansei.ac.jp; Tel./Fax: +81-79-565-7228.
} 
mode has an IR or Raman band at a specific wavenumber corresponding to its transition energy, the molecule therefore has a specific band pattern in the spectrum. The IR and Raman spectra are collectively referred to as the "molecular fingerprint" of a molecule. When a sample consists of relatively small molecules and a simple composition, IR and Raman spectroscopy are powerful techniques to use for the identification of the molecules. Molecular labelling is unnecessary as all of the sample molecules have specific band patterns. In contrast, when the sample includes many complicated materials, such as proteins and DNA, the spectrum is often too complicated to permit the identification or analysis of a specific molecule in the sample. NIR spectroscopy is based on the energy transition between vibrational energy levels, but not energy levels that are neighboring each other as this is generally prohibited. This irregular transition can be possible because of anhamonicity between theoretical (harmonic) and real (anharmonic) oscillations of the molecular vibrations. Since the anhamonicity is very small, the optical absorption in NIR spectroscopy is also very small compared with IR spectroscopy. NIR light has a much higher transmission efficiency in organic materials, from millimeter to centimeters, than that of IR light, which transmits only several micrometers. NIR spectroscopy is often used for nondestructive analysis.

Raman spectroscopy has advantages in the field of biology, especially for observing live samples, compared with IR spectroscopy [13]. Live samples usually contain plenty of water, have an indivisible unit (e.g. cell), and are very sensitive to the environment. In the IR and NIR spectra, the strong, broad absorption band corresponding to water conceals the bands of other organic molecules. In contrast, the Raman spectrum of water reveals a strong, broad band near $3600 \mathrm{~cm}^{-1}$ that does not overlap with those of other materials. The other advantage is the use of visible and near-NIR light from 400 to $1000 \mathrm{~nm}$ wavelengths. It is often necessary to bring the instrument at the site of the observation, especially for live samples. A normal visible light microscope can be used for Raman microscopy. Additionally, a fiberoptic Raman probe permits Raman measurements in human and animal bodies. The usual objective lens of microscope has a high transmission efficiency and the charge coupled device (CCD) detector has high sensitivity for visible and near-NIR light. A general glass optical fiber does not transmit IR light, it is another weak point of IR spectroscopy. The other advantage is that molecules can be identified by Raman spectrum without labelling, as each molecule has a specific band pattern. This advantage replaces the need for staining, fixing, and even killing the live sample. However, there is one drawback. The molecular composition of biological samples are so complicated that the sample spectrum consists of overlapping bands that will conceal the information of a specific molecule. Hence, the knowledge that the Raman spectrum provides is different from that obtained by conventional molecular biology.

Multivariate analysis is often used to evaluate the Raman spectrum and extract useful information $[13,23]$. Since the biological sample has inherent fluctuation, the molecular composition of biological samples shows a certain level of variations. There are a several types of general statistical methods, such as F-test, to analyze the variance of datasets and they are often used in biological analysis. In contrast, multivariate analysis is based on linear-algebra, such as principal component analysis (PCA) and partial least square regression (PLSR) analysis, and it is useful for spectral analysis. We do not discuss chemometrics here because it is out of the scope of the present manuscript. Please refer to review papers and available books on chemometrics [13,23].

\section{Live cell analysis}

Raman spectroscopy provides information on the molecular composition of cells and organelles without any treatment of the sample. A Raman microscope has spatial resolutions as high as an optical 


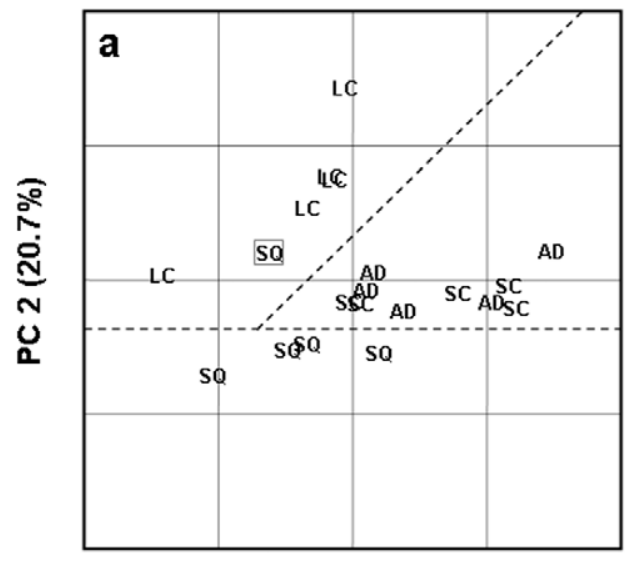

PC $1(44.4 \%)$

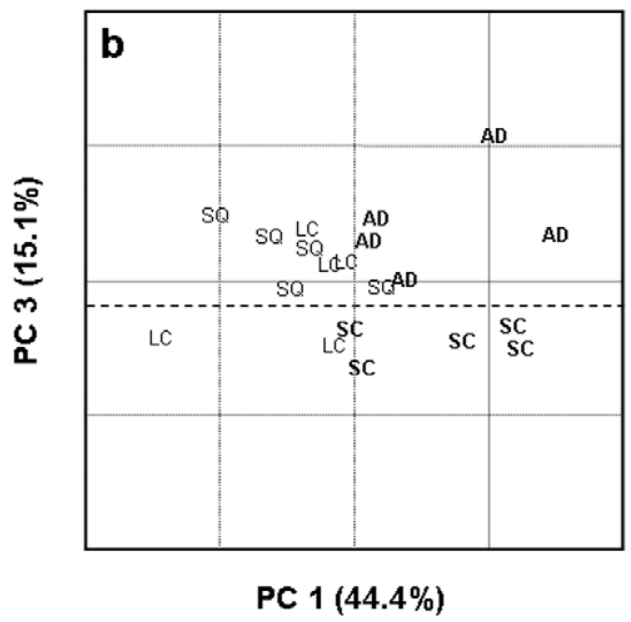

Fig. 1. PCA score plots for PCs 1 and 2 (a) and PCs 1 and 3 (b) of the Raman spectra of normal (CT), adenocarcinoma (AD), squamous cell carcinoma (SQ), large cell carcinoma (LC), and small cell carcinoma (SC). (Reproduced from reference [19].)

microscope. The effects of chemical treatment can also be explored by the nondestructive and rapid monitoring of the cellular condition [4,5,17-19]. Oshima et al. reported that Raman spectroscopy is capable of identifying normal (CT) and different types of human lung cancer cells, such as adenocarcinoma (AD), squamous cell carcinoma (SQ), large cell carcinoma (LC), and small cell carcinoma (SC), cultured in dishes [19]. Figure 1 shows the PCA score plots for PC1-2 (a) and PC1-3 (b). The cells are well-discriminated in the plots, except one data point for SC. Strong bands were observed at 747, 1127, and $1583 \mathrm{~cm}^{-1}$ in their loading plots. The bands were assigned to cytochrome c (cyt-c) since the spectral pattern was similar to the Raman spectrum of reduced and oxidized cyt-c. The loading plot of the PCA indicates that the relative intensities among these bands are the markers for identifying the cells. The bands were observed in the cellular spectrum measured with an excitation of $532 \mathrm{~nm}$ but not in those measured with excitation wavelengths of 785 and $633 \mathrm{~nm}$. It is strongly suggested that the bands were resonance enhanced by Q-bands of cyt-c from 510 to $550 \mathrm{~nm}$ with the excitation of $532 \mathrm{~nm}$.

The molecular composition of the cell changes once there are any changes in cell function. Hashimoto et al. has applied Raman analysis to study the function of neural cells [4,5]. Upon differentiation into a neuron, the neuron cell cycle ceases and it matures to develop neuron function. An electrophysiological study revealed that neurons gradually coordinated to construct a network in a culture dish. It generally takes 10 days for each neuron to make a random electric spike signal, which is referred to as "firing", and 60 days to have synchronized firing over the cellular network. The changes in molecular composition of the maturing neuron were monitored with Raman analysis up to 120 days in the culture dish. The PCA score plots suggest that there are a several steps in neuron growth. The 2 nd day dataset was one independent data group. The second group consisted of datasets from days 8th-30th. The datasets of the 45th-105th day comprised the third group. Then, the dataset of the 120th day was the fourth data group. The dates of the data groups suggested that groups 1-3 represented the maturing steps of the neurons, corresponding to those with no function, random firing, and synchronized firing, respectively. There is no obvious function found for the neurons at the 120th day. The neuron has polarity depending on whether it has an excitatory and inhibitory function. It is quite difficult to identify the neuron polarity by appearance; the major difference is in the type of neurotransmitter. Hashimoto et al. succeeded in using Raman analysis in the completely noninvasive manner to discriminate the excitatory from the inhibitory 
neurons. The Raman spectra were collected from intact neurons in culture dishes, then the polarity of the neurons was confirmed through immunostaining for the inhibitory neurotransmitter $\gamma$-aminobutyric acid (GABA). The reliability of the model built by PLSR-discrimination analysis (DA) was validated using independent test data. The results suggest that the function of the cell closely relates to its molecular composition and demonstrate that Raman spectroscopy permits the study and monitoring of the intact cell function in a completely noninvasive manner.

\section{Raman spectroscopy of live tissue}

The major difference between cell and tissue is the extracellular matrices, including the basal membrane, epithelium, bone, and blood vessels. Although live tissue is a complex system consisting of various cells and extracellular matrices, it is another target that is observed through Raman spectroscopy. To observe live tissue, Raman spectroscopy is often used together with an endoscope. Haka et al. reported the application of Raman endoscopy for analysis of human breast cancer [3]. The results suggested a sensitivity of $83 \%$ and specificity of $93 \%$ for the evaluation of freshly excised surgical specimens. Zheng et al. applied Raman analysis to evaluate single live epithelial cells in colorectal cancer, and they succeeded in discriminating the cancer with $86.3 \%$ sensitivity and $86.3 \%$ specificity [26]. Schulmerich et al. reported the in vivo analysis of bones in mouse using a Raman probe [20].

We have developed two types of narrow Raman probes, and the maximum diameter was less than $650 \mu \mathrm{m}[9-12,16]$. A miniature endoscope for mice was also developed to evaluate the performance of the probes [6]. The use of a mouse model offers several advantages. It is possible to monitor the advancement of a tumor from an early stage. Individual discordance is eliminated because the mouse is isogenic. Taketani et al. employed the miniaturized Raman endoscope to study colorectal cancer advancement in an AOM-DSS mouse model. The endoscope head, which was $2.5 \mathrm{~mm}$ in diameter with a $1.2 \mathrm{~mm}$ diameter channel, was used together with a ball lens-installed hollow optical fiber Raman probe (BHRP) [25]. The endoscopic observation with Raman measurement was carried out at 15, 17, and 19 weeks of age. PLSR score plots for factors 1 and 2 calculated for 3 tumor mice are shown in Fig. 2 [25]. The datasets were classified into three groups according to the number of weeks in the score plot, which indicated that the Raman analysis provided an estimation of tumor progression.

The effects of anticancer drugs were examined in the colorectal mouse model using the Raman endoscope [24]. The mouse models were treated with 5 fluorouracil (5-FU), cisplatin (CDDP), and docetaxel, and the endoscopic observation was carried out after 2 weeks. The PLSR discrimination analysis (PLSADA) successfully detected the effect of the drugs on the colorectal tumor lesions. The results suggest that the drugs are delivered into the tumor tissues where they induce changes at the molecular level. Spectral changes were also observed in the colorectal wall suggesting that the drugs affect normal cells and tissues. The present results do not provide information on whether the molecular changes relates to the therapeutic effect or side effect.

\section{Application study for cancer detection}

For cancer treatment, early detection and rapid cure are the most important factors for maximizing the therapeutic effect. Narrow band imaging (NBI) and auto-fluorescence imaging (AFI) have made breakthroughs in endoscopic diagnostic technology. These techniques successfully emphasize the cancer lesion of gastrointestinal tissues using color contrast and brightness with simple optical principles. 

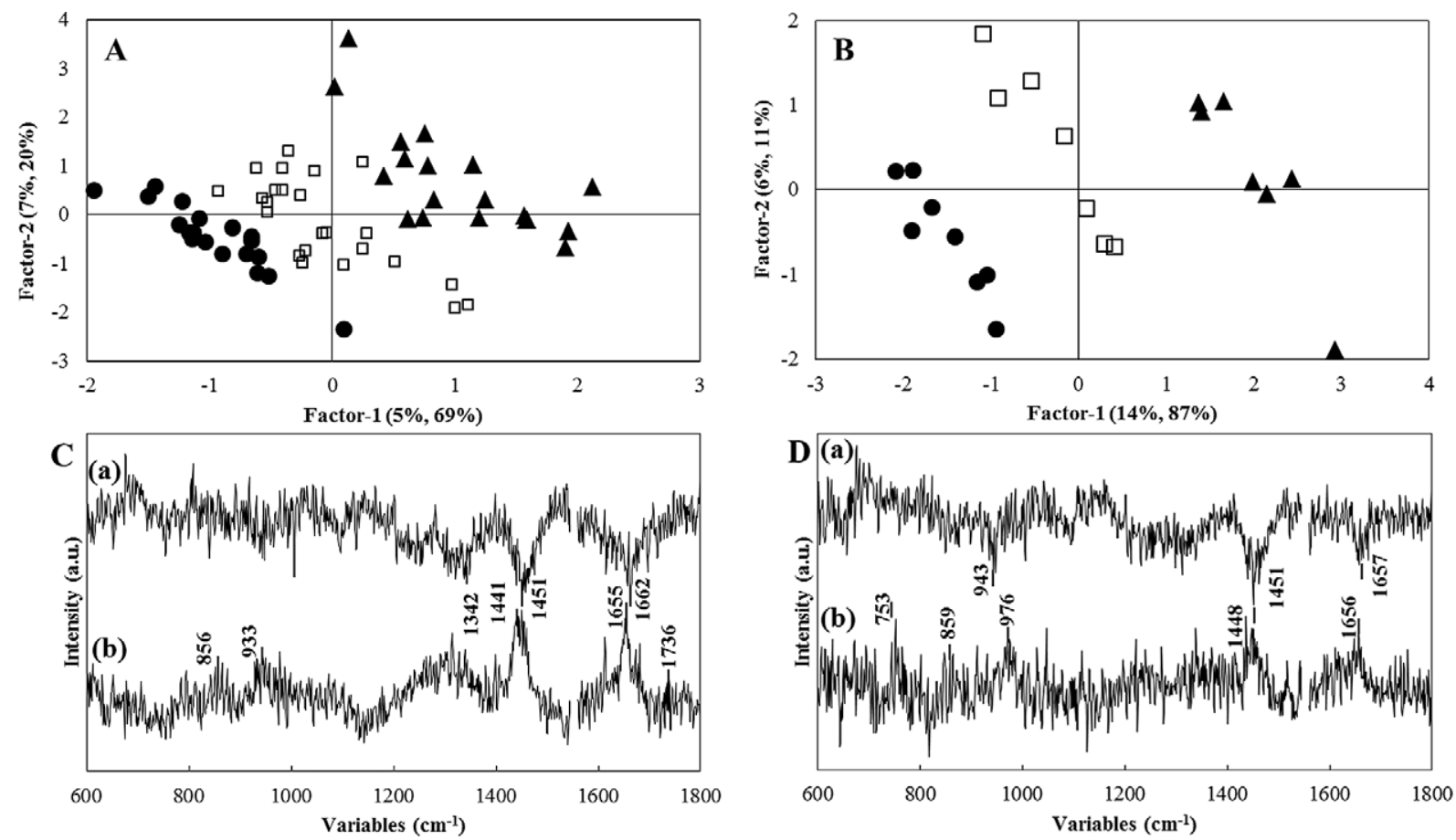

Fig. 2. Score and loading plots for factors 1 and 2 of the PLSR models obtained for the spectra measured in 3 tumor mouse models (A and C) and a single tumor mouse model (B and D) of the symbols $\boldsymbol{\bullet}, \square$, and $\boldsymbol{\Delta}$ represent the spectral data measured at 15,17 , and 19 weeks of age, respectively. The Raman spectra were measured at several tumor lesions in the mouse; for the 3 tumor mouse models, the total number of spectra measured were 19,24, and 19 corresponding to 15, 17, and 19 weeks, respectively; for the single tumor mouse model, the total number of spectra were 8,7 , and 7 for 15,17 , and 19 weeks, respectively. (Reproduced from the reference [25].)

For the future, the development of a medical technique to detect cancers in the very early stage before morphological changes appear is needed; Raman spectroscopy is one of the most powerful tools for this purpose. It enables us to obtain molecular information on the tissue in situ without labeling. In recent years, Raman spectroscopy has been applied to the endoscopic diagnosis of gastric and esophageal cancers $[1,2]$. Huang et al. developed a fiber optic Raman probe with a $1.8 \mathrm{~mm}$ diameter inserted through the forceps of an endoscope [7]. The Raman spectra were obtained within $0.5 \mathrm{~s}$ from in vivo tissue during endoscopic observation using a $785 \mathrm{~nm}$ excitation laser. In many publications, the analytical models built for the detection of cancer had datasets that included many advanced cases that were obviously recognized even by visual observation. Since morphological changes are presumed to occur after cellular molecular changes in concentrations, compositions, and conformations due to cancer metabolism, Raman spectroscopy can detect very early cancer lesions by capturing the signals of these molecular changes. Hence, we tried to discriminate very early human esophagus cancer based on molecular information using Raman spectroscopy [8]. An ex vivo study was conducted using stage 0 and I esophageal cancer tumor tissues. They were surgically collected by endoscopic mucosal resection (EMR) under endoscopic observation. The portable Raman system was set up in the room next to the operation room, and Raman signals were immediately recorded within $1 \mathrm{~h}$ after the tissue resection. After Raman measurement, the tissues were immediately fixed with formalin. The fixed tissues were sent for histopathological examination and the spectroscopic data were analyzed together with the histological results. Raman spectra (stage I $[n=42]$, stage $0[n=25]$, suspicious lesions $[n=6]$, and normal areas $[n=50]$ ) in 


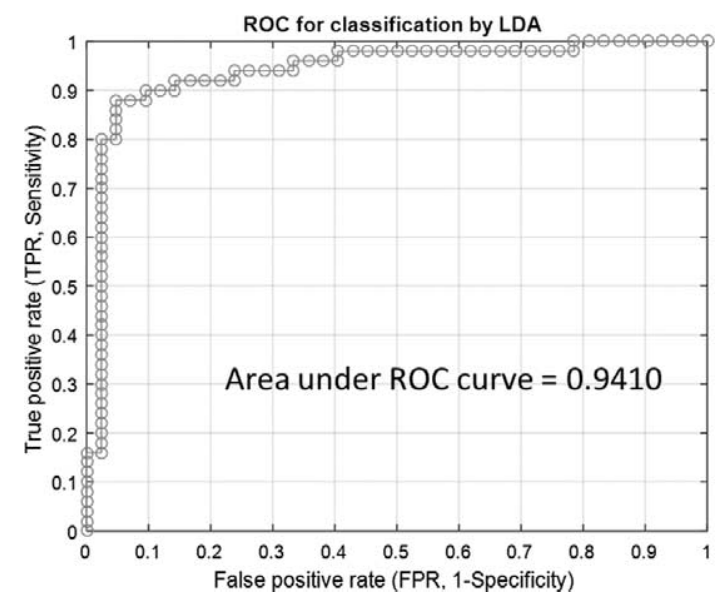

Fig. 3. An ROC curve plotted the proportion of true positives (TPs) against the proportion of false positives (FPs). The LDA diagnostic algorithm validated these two tissue types using the leave-one-out cross-validation (LOOCV) methodology. (Reproduced from reference [8].)

the 1800-600 $\mathrm{cm}^{-1}$ wavenumber region were obtained from the tissues. The definition of a "suspicious lesion" in the study was an area of either normal or cancerous tissue that could not be definitively diagnosed by histological examination; these data were assigned to cancerous ones in the datasets. The unpaired two-sided Student's $t$-test showed that six bands (850, 867, 922, 1038, 1366, and $1627 \mathrm{~cm}^{-1}$ ) were significantly different between cancer and normal tissues. These bands were common to glycogen, collagen, and tryptophan, and their concentrations appeared to be lower in the cancerous tissues. Using these bands, a linear discriminant analysis (LDA) model using leave-one-out cross-validation (LOOCV) was constructed to discriminate each Raman spectrum based on whether it showed a characteristic pattern for cancer versus normal tissue. The fraction of true positive (cancer sample predicted as cancerous tissue) and the false positives (normal sample predicted as cancerous tissue) were calculated. The results are summarized in the receiver operating characteristic (ROC) curve shown in Fig. 3. The sensitivity of and specificity for cancer diagnosis of the present model were $81.0 \%$ and $94.0 \%$, respectively. The results indicate that Raman spectroscopy is able to detect molecular variations occurring in esophageal tissue at a very early stage. Thus, this technique is a strong candidate as practical medical technology to use for in situ early-stage cancer diagnosis prior to the appearance of morphological changes.

\section{Application of health controls: Fat analysis}

A large amount of data has been collected on patients with diseases, but there is very little information on healthy individuals. Recently, researchers have been intent on studying the prediction of disease and are trying to develop techniques for improving the accuracy of preliminary diagnosis. To reduce the expanding medical cost in a society, it is important to apply specific health controls before the occurrence of disease. Big data analysis of human activity is a strong candidate for use in the estimation of the individual health condition as posteriori environment and hereditary factors have been known to largely affect disease development. However, it is difficult to include the molecular information of individuals as the method generally requires invasive measures for the molecular analysis of human tissue. Healthy people usually do not agree to painful tests requiring the cutting of tissue. Consequently, Raman spectroscopy can be used for painless health check-ups as it is a noninvasive technique. 
Meksiarun et al. succeeded in collecting information on subsurface tissue using a painless method with the fiber optic Raman probe [14,15]. Fats are indispensable materials in the human body, but too much fat causes obesity which often correlates to other adult diseases. Fat is synthesized in our body but is also harvested from the food that we eat. Three groups of hamsters were given diets of tricaprin (TC), trilinolein (TL), and normal saline (control), and their adipose tissue accumulations were monitored for several weeks. The fatty chain of TC has 10 carbons and no double bonds whereas the TL fatty chain has 18 carbons and 2 double bonds. The fat compositions in the adipose tissues were analyzed by gas chromatography (GC) and Raman analysis. A fiber optic Raman probe that had $\sim 800 \mu \mathrm{m}$ of working distance was developed especially for the analysis of subcutaneous adipose. The speeds of accumulation were $4.5 \%$ and $0.6 \%$ per 2 weeks for TL and TC, respectively. The results of noninvasive subsurface analysis and of GC and the direct Raman analysis of resected tissues were almost the same, demonstrating that the Raman analysis with the fiber optic probe is highly useful for noninvasive body fat analysis. The results also suggest that the ratio of fat intake depends on the fat species. The painless Raman measurement of subcutaneous adipose can provide a good, objective direction for obesity control and be used to collect information on healthy people.

\section{Conclusion}

The technical developments in the last 20 years have led to the use of Raman spectroscopy for highly sensitive biomedical analysis. The developments in multivariate analysis with Raman spectroscopy has opened up a new era of label-free live cell analysis. Since molecular compositional changes during the cell cycle is relatively small and regulated, cellular modifications can be identified by using the technique. The research results suggest that Raman analysis is a powerful method to use for the differentiation of stem cells as well as the small molecular alterations relating to their function. The development of the fiber optic Raman probe has enabled Raman measurement in the bodies of live animals as well as humans, resulting in the medical and clinical application of the technique. In the health sciences, researchers are very interested in the accurate prediction of disease. Raman spectroscopy also has a high potential for the painless analysis of healthy people.

\section{References}

[1] M.S. Bergholt, W. Zheng, K. Lin, K.Y. Ho, M. Teh, K.G. Yeoh, J.B.Y. So and Z. Huang, Raman endoscopy for in vivo differentiation between benign and malignant ulcers in the stomach, Analyst 135 (2010), 3162-3168. doi:10.1039/ c0an00336k.

[2] M.S. Bergholt, W. Zheng, K. Lin, K.Y. Ho, M. Teh, K.G. Yeoh, J.B.Y. So and Z. Huang, In vivo diagnosis of esophageal cancer using image-guided Raman endoscopy and biomolecular modeling, Technol. Cancer Res. Treat. 10 (2012), 103112. doi:10.7785/tcrt.2012.500185.

[3] A.S. Haka, Z. Volynskaya, J.A. Gardecki, J. Nazemi, R. Shenk, N. Wang, R.R. Dasari, M. Fitzmaurice and M.S. Feld, Diagnosing breast cancer using Raman spectroscopy: Prospective analysis, J. Biomed. Opt. 14 (2009), 054023.

[4] K. Hashimoto, B.B. Andriana, H. Matsuyoshi and H. Sato, Discrimination analysis of excitatory and inhibitory neurons using Raman spectroscopy, Analyst 143 (2018), 2889. doi:10.1039/C8AN00051D.

[5] K. Hashimoto, S.N. Kudoh and H. Sato, Analysis of the developing neural system using an in vitro model by Raman spectroscopy, Analyst 140 (2015), 2344-2349. doi:10.1039/C4AN01961J.

[6] Y. Hattori, Y. Komachi, T. Asakura, T. Shimosegawa, G. Kanai, H. Tashiro and H. Sato, In vivo Raman study of the living rat esophagus and stomach using a micro Raman probe under endoscope, Appl. Spectrosc. 61 (2007), 579-584. doi:10. $1366 / 000370207781269747$. 
[7] Z. Huang, S.K. Teh, W. Zheng, J. Mo, K. Lin, X. Shao, K.Y. Ho, M. Teh and K.G. Yeoh, Integrated Raman spectroscopy and trimodal wide-field imaging techniques for real-time in vivo tissue Raman measurements at endoscopy, Opt. Lett. 34 (2009), 758-760. doi:10.1364/OL.34.000758.

[8] M. Ishigaki, Y. Maeda, A. Taketani, B.B. Andriana, R. Ishihara, K. Wongravee, Y. Ozaki and H. Sato, Diagnosis of earlystage esophageal cancer by Raman spectroscopy and chemometric techniques, Analyst 141 (2016), 1027-1033. doi:10. 1039/C5AN01323B.

[9] T. Katagiri, Y.S. Yamamoto, Y. Ozaki, Y. Matsuura and H. Sato, High axial resolution Raman probe made of single hollow optical fiber, Appl. Spectrosc. 63 (2009), 103-107. doi:10.1366/000370209787169650.

[10] Y. Komachi, H. Sato, K. Aizawa and H. Tashiro, Micro optical fiber probe for the use of intravascular Raman endoscope, Appl. Opt. 44 (2005), 4722-4732. doi:10.1364/AO.44.004722.

[11] Y. Komachi, H. Sato, Y. Matsuura, M. Miyagi and H. Tashiro, Raman probe using a single hollow waveguide, Opt. Lett. 30 (2005), 2942-2944. doi:10.1364/OL.30.002942.

[12] Y. Komachi, H. Sato and H. Tashiro, Intravascular Raman spectroscopic catheter for molecular diagnosis of atherosclerotic coronary disease, Appl. Opt. 45 (2006), 7938-7943. doi:10.1364/AO.45.007938.

[13] P. Matousek and M.D. Morris (eds), Emerging Raman Applications and Techniques in Biomedical and Pharmaceutical Fields, Springer, Berlin, Heidelberg, 2010.

[14] P. Meksiarun, B.B. Andriana, H. Matsuyoshi and H. Sato, Non-invasive quantitative analysis of specific fat accumulation in subcutaneous adipose tissues using Raman spectroscopy, Sci. Rep. 6 (2016), 37068.

[15] P. Meksiarun, N. Spegazzini, H. Matsui, K. Nakajima, Y. Matsuda and H. Sato, In vivo study of lipid accumulation in the microalgae marine diatom Thalassiosira pseudonana using Raman spectroscopy, Appl. Spectrosc. 69 (2015), 45-51. doi:10.1366/14-07598.

[16] H. Mitsuoka, S. Morita, T. Suzuki, Y. Matsuura, Y. Katsumoto and H. Sato, Optical characterization of a hollow fiber Raman probe toward non-invasive measurements of living tissues, Appl. Phys. Exp. 2 (2009), 027001.

[17] K. Moor, K. Ohtani, D. Myrzakozha, O. Zhanserkenova, B.B. Andriana and H. Sato, Noninvasive and label-free determination of virus infected cells by Raman spectroscopy, J. Biomed. Opt. 19 (2014), 067003.

[18] K. Moor, Y. Terada, A. Taketani, H. Matsuyoshi, K. Ohtani and H. Sato, Early detection of virus infection in live human cells using Raman spectroscopy, J. Biomed. Opt. 23 (2018), 097001.

[19] Y. Oshima, H. Shinzawa, T. Takenaka, C. Furihata and H. Sato, Discrimination analysis of human lung cancer cells associated with histological type and malignancy using Raman spectroscopy, J. Biomed. Opt. 15 (2010), 017009.

[20] M.V. Schulmerich, J.H. Cole, J.M. Kreider, F.E. White, K.A. Dooley, S.A. Goldstein and M.D. Morris, Transcutaneous Raman spectroscopy of murine bone in vivo, Appl. Spectrosc. 63 (2009), 286-295. doi:10.1366/000370209787599013.

[21] N.C. Shaner, M.Z. Lin, M.R. McKeown, P.A. Steinbach, K.L. Hazelwood, M.W. Davidson and R.Y. Tsien, Improving the photostability of bright monomeric orange and red fluorescent proteins, Nature Methods 5 (2008), 545-551. doi:10.1038/ nmeth.1209.

[22] O. Shimomura, The discovery of aequorin and green fluorescent protein, J. Microsc. 217 (2005), 3-15. doi:10.1111/j. 0022-2720.2005.01441.x.

[23] H.W. Siesler, Y. Ozaki, S. Kawata and H.M. Heise (eds), Near-Infrared Spectroscopy: Principles, Instruments, Applications, Wiley-VCH, Weinheim, 2002.

[24] A. Taketani, B.B. Andriana, H. Matsuyoshi and H. Sato, Raman endoscopy for monitoring the anticancer drug treatment of colorectal tumors in live mice, Analyst 142 (2017), 3680-3688. doi:10.1039/C7AN00720E.

[25] A. Taketani, R. Hariyani, M. Ishigaki, B.B. Andriana and H. Sato, Raman endoscopy for the in situ investigation of advancing colorectal tumors in live model mice, Analyst 138 (2013), 4183-4190. doi:10.1039/c3an00169e.

[26] F. Zheng, Y. Qin and K. Chen, Sensitivity map of laser tweezers Raman spectroscopy for single-cell analysis of colorectal cancer, J. Biomed. Opt. 12 (2007), 034002. 